\title{
Facial EMG Activity Levels Predict Treatment Outcome in Depression
}

\author{
John F. Greden, H. Laurence Price, Nancy Genero, Michael Feinberg, and \\ Simon Levine
}

Received February 21, 1984; revised version received July 13, 1984; accepted August 5, 1984.

\begin{abstract}
Higher electromyographic (EMG) activity levels of corrugator and zygomatic face muscles have been reported to be pretreatment predictors of better clinical outcome in depressed patients. We tested this possibility in $29 \mathrm{drug}$-free, rigorously diagnosed subjects by measuring low-level EMG activity of corrugator and zygomatic muscles during resting and three imagery states (happy, typical day, sad). All patients had major depressive disorder, endogenous subtype. Good responders had significantly higher pretreatment EMG zygomatic values and different EMG profiles. Our findings replicate and expand prior reports.
\end{abstract}

Key Words. Facial electromyography, corrugator muscles, zygomatic muscles, depression, outcome.

Facial expressions are important barometers of psychomotor state in depressed patients (Greden and Carroll, 1981). The musculature of the face responds to emotional changes with unique speed and sensitivity (Ekman and Friesen, 1975), and the facial expressions associated with happiness, sadness, anger, fear, surprise, and disgust are believed to be universal (Darwin, 1872; Ekman et al., 1969). The corrugator muscles, located on the forehead bilaterally above the bridge of the nose and referred to by Darwin (1872) as the "grief muscles," are considered especially important in displays of sadness, mourning, and grief (Ekman and Friesen, 1972). Although facial expressions provide visible windows to affective states, their assessment has been subjective and error-prone (Whatmore and Ellis, 1975; Ekman and Friesen, 1975; Greden and Carroll, 1981).

In an attempt to develop an objective, quantifiable monitor for facial activity, Schwartz et al. $(1975,1976 a, 1976 b, 1978,1979,1980)$ studied the use of electromyographic (EMG) measures of low-level facial muscle contractions. He and colleagues placed surface electrodes upon selected muscle regions of the face and recorded EMG activity during self-induced states of affective imagery (e.g., happy,

John F. Greden, M.D., is Professor of Psychiatry, Director, Clinical Studies Unit for Affective Disorders, and Scientific Associate. Mental Health Research Institute. University of Michigan Hospitals. H. Laurence Price, M.S., and Nancy Genero, M.A., are in the Clinical Studies Unit. Department of Psychiatry, University of Michigan Hospitals. Michael Feinberg, M.D., Ph.D., is Associate Professor, Department of Psychiatry and Mental Health Research Institute, University of Michigan Hospitals. Simon Levine, Ph.D., is in the Department of Physical Medicine and Rehabilitation. University of Michigan, Ann Arbor, MI. (Reprint requests to Dr. J.F. Greden, Clinical Studies Unit for Affective Disorders, Dept. of Psychiatry, University of Michigan Hospital, N5737, Ann Arbor, MI 48109. USA.) 
sad, angry, and "typical day"). A number of muscle regions were studied: (1) corrugator (located bilaterally on the forehead above the bridge of the nose); (2) zygomatic, the "smile muscle," located between the angles of the mouth and cheekbones bilaterally); and (3) peri-oral muscles such as the depressor anguli oris, depressor labii inferioris, and the mentalis (these muscles produce changes in mouth expressions during affective fluctuations). Using their EMG approach, Schwartz et al. found diagnostic differences between depressives and normals. Each discrete imagery task produced a distinct EMG profile (Schwartz et al., 1976a, 1976b), and depressed patients differed from nondepressed controls on the basis of their profiles. These results have been replicated by other investigational teams (Teasdale and Bancroft, 1977; Oliveau and Willmuth, 1979; Greden et al., in press).

Perhaps of greater interest than possible diagnostic differences, Schwartz et al. (1978) reported that higher baseline corrugator EMG activity seemed to predict better clinical outcome in their depressed patients. Specifically, they noted that those subjects with the highest initial corrugator levels showed the greatest clinical improvement regardless of treatment selected (amitriptyline or placebo). Laboratory predictors of treatment response are such potentially powerful tools (consider the benefits of bacterial culture and sensitivity testing for selecting antibiotics in patients with infectious diseases) that attempts at replication were predictable. Carney et al. (1981) confirmed that subjects with higher initial corrugator EMG levels had greater changes in depressive symptoms, and suggested that resting zygomatic EMG scores also predicted good outcome.

These studies on prediction of outcome were pilot assessments and subject to expected methodological shortcomings. Specifically, Schwartz and colleagues studied: (1) only outpatients; (2) the investigations were not conducted under fully "blind" conditions; (3) they required only an unstructured clinical diagnosis of depression and a Zung score above 56 as their inclusion criteria; (4) they studied only the corrugator at rest; (5) they treated patients with relatively low doses of amitriptyline (75-150 mg) with no monitoring of plasma levels; and (6) they combined a sample of 5 males and 14 females, later reported by the same group to have different facial EMG responses (Schwartz et al., 1980). Carney et al. (1981) studied only female inpatients with significant unipolar depression (Beck depression scores above 20), and studied three left-sided muscle regions; however, 17 or their 21 subjects had been receiving tricyclic antidepressants before study. Both studies also were limited to one followup assessment at 2 weeks, and both emphasized changes in rating scale scores as dependent variables of improvement, thus introducing possible contamination due to Wilder's (1957) law of initial values (subjects with higher levels at one time point will show greater decreases at a second time point). Schwartz et al. (1978) stated: "Until the specific finding for resting corrugator activity is replicated on a larger sample with full-blind conditions, it must be seen as tentative." We agreed, and sought to control for methodological issues mentioned above. After doing so, we replicated and expanded earlier reports that pretreatment EMG activity levels may predict treatment outcome. 


\section{Methods}

Subjects, Setting, and Outcome Criteria. We studied 29 right-handed, female, nondelusional patients. Each was hospitalized in the Clinical Studies Unit (CSU) for Affective Disorders, a 12-bed unit at the University of Michigan Hospital. Each patient was drug-free for 10-14 days before EMG testing. Our evaluation procedure has been described in detail elsewhere (Greden et al., 1980). In summary, each patient underwent a detailed assessment after drug washout. The assessment included two independent clinical and structured interviews (Schedule for Affective Disorders and Schizophrenia; SADS) (Spitzer and Endicott, 1975) and thorough medical screening. Treatments were selected for clinical efficacy. All subjects were treated with imipramine, desipramine, amitriptyline, or maprotoline. Plasma concentrations were documented to be in the therapeutic range $(>180 \mathrm{ng} / \mathrm{ml})$ for 27 of the 29 patients. Two individuals received maprotoline in an accepted dose range, but had no plasma assays (both were good responders). All diagnoses and treatment selections were formulated by diagnosticians unaware of EMG results. Raters without knowledge of EMG data completed the 17-item Hamilton Rating Scale for Depression (HRSD) (Hamilton, 1960) on a weekly basis.

Good responders were defined before data analysis as patients with at least a $50 \%$ decrease in HRSD scores and a final HRSD score of less than 10 (to account for the law of initial values). Poor responders were patients with one or neither of these two criteria.

As shown in Table 1, good responders and poor responders did not differ significantly in age, pretreatment severity, diagnostic subtype (unipolar vs. bipolar), postdexamethasone plasma cortisol concentration, or length of treatment. Because of our outcome criteria, the two subgroups showed predictable posttreatment differences at time of hospital discharge in total HRSD scores, Carroll Depression Scale scores, degree of psychomotor retardation, and maximum postdexamethasone plasma cortisol concentrations.

EMG Techniques. Our EMG procedure, adapted from techniques developed by Schwartz et al., was similar for each subject studied. All patients were asked to complete a questionnaire to detcrmine handedness. EMGs were conducted between 9 and 11 a.m. to control for possible chronobiological factors. Beckman miniature $\mathrm{Ag} / \mathrm{AgCl}$ electrodes were placed bilaterally over the regions of the corrugator and zygomatic muscles. Before electrodes were attached, all skin locations were gently abraded with alcohol pads until a slight redness appeared. Placement procedures were standardized for each muscle region. To distract attention from the study's objectives, we used "dummy" electrodes on both wrists, both forearms over the region of the flexor muscles, and below the left clavicle.

To reduce static charge effects, we placed a single ground connection to the nape of the neck. The low level DC bioelectric potential was amplified, full-wave rectified, and integrated by a Grass Instruments wide band AC preamplifier and integrator (Model 7P3A). This signal was then further amplified by a Grass DC driver amplifier (Model 7DAB). All signals were integrated over a time constant of $500 \mathrm{~ms}$.

Within each session, we stored data collection "runs" for resting (baseline) levels and three imagery states (happy, typical day, and sad). To generate these imagery states, we asked subjects to sit as quietly as possible with eyes closed. We then instructed them: "During this procedure, you will be asked to think about several types of situations. Do the best you can with each of these instructions without actually talking to me. You do not need to tell me what you are thinking. Please try to continue to think about the particular situation until you hear me say to relax."

The specific instructions for the various self-generated stimuli were: "Now I want you to think about a particular situation which made you feel [e.g., happy, sad]. While you are thinking about it, please try to reexperience the feeling you had at that time." Instructions for the typical day imagery were further amplified by telling subjects that we wanted them to think sequentially about what they did throughout a typical day until they went to sleep. 
Table 1. Comparison of demographic and clinical variables in endogenous depressives with good response vs. poor response

\begin{tabular}{|c|c|c|c|c|}
\hline Variables & $\begin{array}{l}\text { Good } \\
\text { responders } \\
(n=20)\end{array}$ & $\begin{array}{l}\text { Poor } \\
\text { responders } \\
(n=9)\end{array}$ & $t$ & $p$ \\
\hline Age & $56.07 \pm 18.49$ & $53.89 \pm 13.60$ & 0.31 & 0.76 \\
\hline \multicolumn{5}{|l|}{ Total 17 -item HRSD (mean $\pm S D)$} \\
\hline Pretreatment & $22.55 \pm 5.79$ & $21.12 \pm 4.61$ & 0.62 & 0.54 \\
\hline Discharge & $3.60 \pm 2.66$ & $17.25 \pm 8.72$ & -6.44 & 0.0011 \\
\hline \multicolumn{5}{|l|}{$\begin{array}{l}\text { Total Carroll depression score } \\
\text { (mean } \pm \text { SD) }\end{array}$} \\
\hline Pretreatment & $33.40 \pm 7.30$ & $30.75 \pm 9.35$ & 0.80 & -0.43 \\
\hline Discharge & $9.73 \pm 6.08$ & $25.25 \pm 11.64$ & -4.57 & 0.0011 \\
\hline \multicolumn{5}{|l|}{$\begin{array}{l}\text { Selected Hamilton Scale items } \\
(\text { mean } \pm \text { SD })\end{array}$} \\
\hline Pretreatment agitation & $0.95 \pm 1.14$ & $0.75 \pm 0.88$ & 0.44 & 0.66 \\
\hline Discharge agitation & $0.22 \pm 0.41$ & $0.62 \pm 0.84$ & -1.55 & $5 \quad 0.13$ \\
\hline Pretreatment retardation & $1.20 \pm 0.83$ & $1.38 \pm 0.91$ & -0.49 & 0.62 \\
\hline Discharge retardation & $0.06 \pm 0.25$ & $1.00 \pm 1.04$ & -3.64 & $4 \quad 0.0011$ \\
\hline \multicolumn{5}{|c|}{$\begin{array}{l}\text { Dexamethasone suppression test (DST) results } \\
(\text { mean } \pm \text { SD) }\end{array}$} \\
\hline $\begin{array}{l}\text { Pretreatment maximum postdexamethas } \\
\text { plasma cortisol }(\mu \mathrm{g} / \mathrm{d} \mid)\end{array}$ & $10.33 \pm 8.77$ & $10.66 \pm 8.94$ & -0.90 & 0.93 \\
\hline $\begin{array}{l}\text { Posttreatment maximum post } \\
\text { dexamethasone plasma cortisol }\{\mu \mathrm{g} / \mathrm{dl} \text { ? } \\
\text { Pretreatment number of DST }\end{array}$ & $3.99 \pm 4.64$ & $9.37 \pm 8.42$ & -2.05 & $5 \quad 0.051$ \\
\hline nonsuppressors $(\%)$ & $14(70 \%)$ & $5(56 \%)$ & & $\begin{array}{l}\chi^{2}=0.112 \\
d f=1 \\
p=0.74\end{array}$ \\
\hline $\begin{array}{l}\text { Posttreatment number of DST } \\
\text { nonsuppressors }(\%)\end{array}$ & $6(30 \%)$ & $5(56 \%)$ & & $\begin{array}{l}\chi^{2}=0.81 \\
d f=1 \\
p=0.37\end{array}$ \\
\hline \multicolumn{5}{|l|}{ Diagnostic subtype } \\
\hline Unipolar & 14 & 6 & & $x^{2}=0.06$ \\
\hline Bipolar & 6 & 3 & & $\begin{array}{l}d f=1 \\
\rho-0.37\end{array}$ \\
\hline
\end{tabular}

1. Statistically significant difference.

Data Analysis. We log-transformed EMG values before statistical analysis to obtain normality of distribution (Gaddum, 1945). We then compared EMG values for good and poor responders using a $2 \times 4$ repeated measures analysis of variance (ANOVA). I he factors used were groups (good and poor responders) and conditions (baseline, happy, typical day, and sad). The $\chi^{2}$ test or Fisher's Exact Probability Test was used to compare nonparametric variables. 


\section{Results}

Group means and standard deviations for each muscle region and condition are presented in Table 2. Fig. 1 depicts pretreatment mean EMG activity values, and compares good and poor responders.

Table 2. Comparison of EMG values in microvolts in left corrugator and left zygomatic regions between good responders and poor responders 1

\begin{tabular}{|c|c|c|c|c|}
\hline & \multicolumn{2}{|c|}{$\begin{array}{l}\text { Patients with major depressive } \\
\text { disorder, endogenous subtype }\end{array}$} & \multirow{2}{*}{\multicolumn{2}{|c|}{$\begin{array}{c}\text { Student's } \\
\text { two-sample } \\
\text { t test }\end{array}$}} \\
\hline & \multirow{2}{*}{$\begin{array}{l}\text { Good responders } \\
(n=20)\end{array}$} & \multirow{2}{*}{$\begin{array}{l}\text { Poor responders } \\
(n=9)\end{array}$} & & \\
\hline & & & $t$ & $\rho$ \\
\hline \multicolumn{5}{|l|}{ Left corrugator } \\
\hline Baseline & $3.17 \pm 0.96$ & $3.17 \pm 0.75$ & -0.02 & 0.99 \\
\hline Happy & $3.37 \pm 0.95$ & $3.09 \pm 0.84$ & 0.73 & 0.47 \\
\hline Typical day & $3.39 \pm 0.90$ & $3.11 \pm 0.77$ & 0.79 & 0.43 \\
\hline Sad & $3.63 \pm 0.95$ & $3.43 \pm 0.63$ & 0.56 & 0.57 \\
\hline \multicolumn{5}{|l|}{ Left zygomatic } \\
\hline Baseline & $2.81 \pm 0.65$ & $2.37 \pm 0.37$ & 1.85 & 0.07 \\
\hline Happy & $2.89 \pm 0.59$ & $2.35 \pm 0.34$ & 2.43 & 0.022 \\
\hline Typical day & $2.83 \pm 0.58$ & $2.37 \pm 0.39$ & 2.13 & 0.042 \\
\hline Sad & $2.89 \pm 0.64$ & $2.49 \pm 0.40$ & 2.48 & 0.10 \\
\hline
\end{tabular}

1. Results are presented as mean \pm SD.

2. Statistically significant difference.

To analyze facial muscle patterns and group differences, we conducted a $2 \times 4$ repeated measures ANOVA (group as the between factor and condition as the within factor) for the zygomatic and corrugator muscle regions. For the zygomatic region, we found a significant main effect for group $(F=5.09 ; d f=1,27 ; p<0.05)$. EMG activity for the good responders was generally higher across conditions than that for the poor responders. No main effect for condition was found $(F=2.07 ; d f=3,81 ; p>0.05)$, indicating similar EMG activity across conditions within each group. No group $\times$ condition interaction was found $(F=0.78 ; d f=3,81 ; p>0.05)$.

We then conducted a repeated measures ANOVA for the corrugator muscle region. In contrast to the zygomatic region, no significant main effect was found for group $(F$ $=0.30 ; d f=1,27 ; p>0.05)$. A significant main effect for condition was noted $(F=5.99$; $d f=3,81 ; p<0.001)$. No group $\times$ condition interaction was found $(F=1.02 ; d f=3,81 ; p$ $>0.05$ ). Thus, alt hough no significant group differences were found between good and poor responders in the corrugator region, the condition main effect suggests that both good and poor responders have greater EMG activity for sad imagery than for happy and typical day imagery. Differences across the three imagery states are illustrated in Fig. 1 .

\section{Discussion}

These data support prior findings (Schwartz et al., 1978; Carney et al., 1981) that pretreatment facial EMG values may predict treatment outcome in endogenous depressives treated with antidepressants. 
Fig. 1. Pretreatment electromyographic (EMG) activity levels during baseline and three imagery states (happy, typical day, and sad) in depressed patients

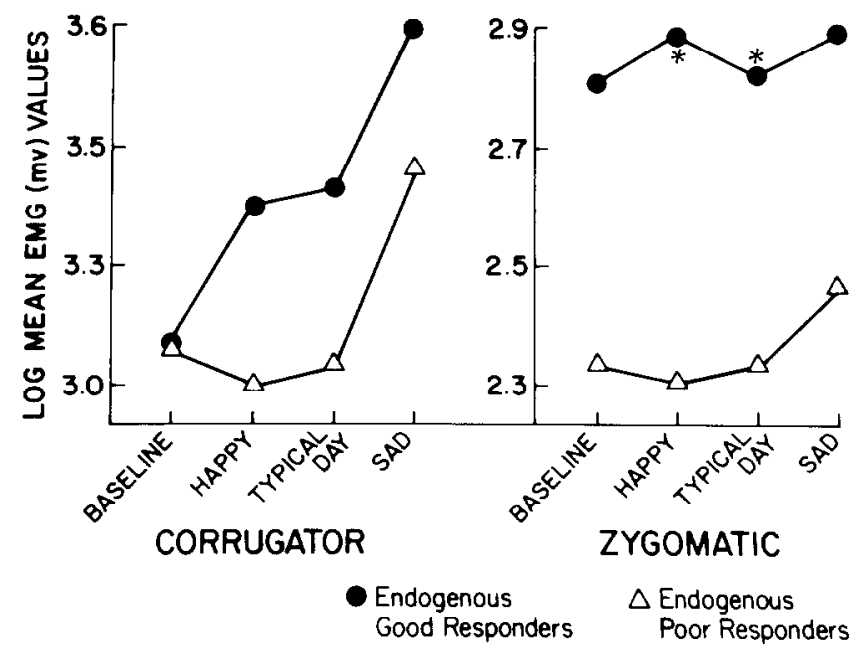

Patients $(n=29$ ) all had major depressive disorder, endogenous subtype. Solid circles show log-transformed EMG values for good responders $(n=20)$, and open triangles show values for poor responders $n=9$ ). Asterisks reveal significant differences based on Student's two-sample $t$ test.

It is not surprising that an electrophysiological measure of psychomotor state might be linked to treatment response because the psychomotor variables of agitation and retardation have historically been considered important clinical predictors of treatment outcome (Overall et al., 1966; Paykel, 1972; Paykel et al., 1973; Kupfer and Spiker, 1981). Possible explanations for the ability of pretreatment EMG activity to predict clinical outcome include the following:

1. EMG values might reflect heterogeneity among endogenous depressives, with differing subgroups having differing outcomes. For example, if one subgroup of depressives had increased psychophysiological "arousal" and consequently increased EMG levels, and if, moreover, the central nervous system (CNS) pathophysiology producing such arousal was responsive to antidepressants, then increased EMG levels might predict better response. Conversely, a different subgroup might have CNS psychophysiological "blunting," and the pathophysiology underlying this state might be unresponsive or less responsive to traditional antidepressants. These prospects have credibility, since close associations between CNS activity and facial expressions have been demonstrated, with voluntary creation of facial prototypes of emotion having been shown to generate immediate emotion-specific activity in the autonomic nervous system (Ekman et al., 1983).

2. EMG values might covary with other factors that influence outcome, such as chronicity, age, severity, or presence of delusions. In our study, we found little support for age, severity, or delusions accounting for differences, since good responders and poor responders had no pretreatment differences for age or severity, and we excluded delusional subjects. Chronicity will require further study. 
3. Patients with increased EMG activity (the good responders) might be more "reactive" to the laboratory situation (Schwartz et al., 1978) and the imagery stimuli. Such increased reactivity could be associated with better outcome. This viewpoint is neither supported by prior studies (Schwartz et al., 1978; Carney et al., 1981), since significant differences have been found for baseline values, nor by our study, in which zygomatic baseline differences between good and poor responders approached significance $(p=0.07)$. Nevertheless, the issue requires further evaluation.

4. Patients with low EMG values might have a different etiology of their depressive symptoms-one conceivably associated with a poorer response to antidepressants. For example, could patients with low EMG levels have a pituitary microadenoma with unrecognized Cushing's syndrome and associated depression? Or some other pathophysiologic process not responsive to antidepressant medications?

More replications of these data in studies using broader patient samples are required. Because of our intentional sampling restrictions, we cannot generalize our findings to males, left-handed individuals, or delusional depressives. Studies with electroconvulsive treatments would also be informative to generalize to different treatment modalities. If findings remain robust, attention then can be focused on the scarch for pathophysiologic and/or treatment differences between good responders and poor responders. Clinically, more intense serial monitoring of "high-risk" subjects (those not predicted to have good outcome) could be conducted during treatment, other etiologies could be sought, and treatment adequacy could be monitored and modified if necessary. The ultimate aim would be to identify more specific or effective treatment approaches for patients who currently respond poorly. Thus, facial EMGs conceivably could be integrated with serial monitoring (Kupfer and Spiker, 1981) to produce improved treatment, the sine qua non of any good laboratory test.

Acknowledgments. The authors acknowledge the contributions of Clinical Studies Unit staff, especially Nancy Barber, Barbara Stoutermine, and Virginia Weinberg, Research Nurses; Pamela Flegel, Research Associate; Judy Matthews, Research Assistant; and Kenneth Guire, Statistician.

The research reported was supported by NIMH grant MH-32736 and by the Mental Health Research Institute, University of Michigan.

\section{References}

Carney, R.M., Hong, B.A., O'Connell, M.F., and Amado, H. Facial electromyography as a predictor of treatment outcome in depression. British Journal of Psychiatry, 138, 485 (1981).

Darwin, C. The Expression of the Emotions in Man and Animals. Murray, London (1872).

Ekman, P., and Friesen, W.V. Unmasking the Face. Prentice-Hall, Englewood Cliffs, NJ (1975).

Ekman, P., Friesen, W.V., and Ellsworth, P. Emotion in the Human Face. Pergamon Press, New York (1972).

Ekman, P., Levenson, R.W., and Friesen, W.V. Autonomic nervous system activity distinguishes among emotions. Science, 221, 1208 (1983).

Ekman, P., Sorenson, E.R., and Friesen, W.V. Pan-cultural elements in facial displays of emotion. Science, 164, 86 (1969).

Gaddum. J.H. Log-normal distributions. Nature, 156, 463 (1945).

Greden, J., Albala, A., Haskett, R.F., James, N.McI., Goodman, L., Steiner, M., and 
Carroll, B.J. Normalization of dexamethasone suppression test: A laboratory index of recovery from endogenous depression. Biological Psychiatry, 15, 449 (1980).

Greden, J.F., and Carroll, B.J. Psychomotor function in affective disorders: An overview of new monitoring techniques. American Journal of Psychiatry, 138, I441 (1981).

Greden, J.F., Price, H.L., Genero, N., Feinberg, M., and Levine, S. Facial electromyography in depression: Subgroup differences. Archives of General Psychiatry (in press).

Hamilton, M. A rating scale for depression. Journal of Neurology, Neurosurgery and Psychiatry, 23, 56 (1960).

Kupfer, D.J., and Spiker, D.G. Refractory depression: Prediction of non-response by clinical indicators. Journal of Clinical Psychiatry, 42, 307 (1981).

Oliveau, D., and Willmuth, R. Facial muscle electromyography in depressed and nondepressed hos pitalized subjects: A partial replication. American Journal of Psychiatry, 136, 548 (1979).

Overall, J.E., Hollister, L.E., Johnson, M., et al. Nosology of depression and differential response to drugs. Journal of the American Medical Association, 195, 946 (1966).

Paykel, E.S. Depressive typologies and response to amitriptyline. British Journal of Psychiatry, 120, 147 (1972).

Paykel, E.S., Prusoff, B.A., Klerman, G.L., et al. Clinical response to amitriptyline among depressed women. Journal of Nervous and Mental Disease, 156, 149 (1973).

Schwartz, G.E., Ahern, G.L., and Brown, S.L. Lateralized facial muscle response to positive and negative emotional stimuli. Psychophysiolog.v. 16, 561 (1979).

Schwartz, G.E., Brown, S.L., and Ahern, G.L. Facial muscle patterning and subjective experience during affective imagery: Sex differences. Psychophysiology, 17, 75 (1980).

Schwartz, G.E., Davidson, R.J., and Maer, F. Right hemisphere lateralization for emotion in the human brain: Interactions with cognition. Science, 190, 286 (1975).

Schwartz, G.E., Fair, P.L., Mandel, M.R., Slat, P., Mieske, R.N., and Klerman, G.L. Facial electromyography in the assessment of improvement in depression. Psychosomatic Medicine, 40, 355 (1978).

Schwartz, G.E., Fair, P.L., Slat, P., Mandel, M.R., and Klerman, G.L. Facial expression and imagery in depression: An electromyographic study. Psychosomatic Medicine, 38, 337 (1976a).

Schwartz, G.E., Fair, P.L., Slat, P., Mandel, M.R., and Klerman, G.L. Facial muscle patterning to affective imagery in depressed and nondepressed subjects. Science, 192, 489 (1976b).

Spitzer, R.L., and Endicott, J. Schedule for Affective Disorders and Schizophrenia (SADS). 2nd ed. New York State Psychiatric Institute, New York (1975).

Teasdale, J.D., and Bancroft, J. Manipulation of thought content as a determinant of mood and corrugator electromyographic activity in depressed patients. Journal of Abnormal Psycholugy, 86, 235 (1977).

Whatmore, G.B., and Ellis, R.M. Some neurophysiologic aspects of depressed states: An electromyographic study. Archives of General Psychiatry, 1, 70 (1959).

Wilder, $\mathbf{J}$. The law of initial values in neurology and psychiatry: Facts and problems. Journal of Nervous and Mental Disease, 125, 73 (1957). 